\title{
Alternative Fixation of an Active Middle Ear Implant at the Short Incus Process
}

\author{
Sebastian P. Schraven ${ }^{a}$ Ernst Dalhoff ${ }^{b}$ Daniela Wildenstein ${ }^{b}$ Rudolf Hagen ${ }^{a}$ \\ Anthony W. Gummer ${ }^{\text {b }}$ Robert Mlynski ${ }^{a}$ \\ aDepartment of Oto-Rhino-Laryngology, Plastic, Aesthetic and Reconstructive Head and Neck Surgery, \\ Comprehensive Hearing Center, University of Würzburg, Würzburg, and 'b Department of Otolaryngology, \\ Section of Physiological Acoustics and Communication, University of Tübingen, Tübingen, Germany
}

\section{Key Words}

Middle-ear surgery · Active middle-ear implant · Floating mass transducer · Incus · Laser Doppler vibrometer

\begin{abstract}
Introduction: Since 1996, the preferred approach for positioning the active middle-ear implant Vibrant Soundbridge ${ }^{\odot}$ is a mastoidectomy and a posterior tympanotomy. With this device, placement of the floating mass transducer (FMT) on the long incus process is the standard method for treatment of mild-to-severe sensorineural hearing loss in the case of normal middle-ear anatomy. The aim of this study was to determine the vibrational effectiveness of FMT placement at the short incus process. Materials and Methods: An extended antrotomy and a posterior tympanotomy were performed in 5 fresh human temporal bones. As a control for normal middle-ear function, the tympanic membrane was stimulated acoustically and the vibration of the stapes footplate and the round-window (RW) membrane were (sequentially) measured by laser Doppler vibrometry. Vibration responses for coupling of an FMT to the long incus process (standard coupling) were compared to those for coupling to the short incus process. Results: Apart from narrow frequency bands near 3 and $9 \mathrm{kHz}$ for the stapes footplate and RW membrane, respectively, the velocity responses presented
\end{abstract}

no significant differences between standard coupling of the FMT and coupling to the short incus process. Conclusion: Coupling the FMT to the short incus process may be a viable alternative in cases where the surgical approach is limited to an extended antrotomy. A reliable technique for attachment to the short incus process has yet to be developed.

(c) 2013 S. Karger AG, Base

\section{Introduction}

The Vibrant Soundbridge ${ }^{\odot}$ (VSB) is an active middleear implant for patients with sensorineural, conductive and mixed hearing losses and/or ear canal problems. Following its first implantation in 1996 [Fisch et al., 2001], the VSB has shown safe and effective performance in patients with mild-to-severe hearing loss and has proven to be an alternative to conventional hearing aids [Todt et al., 2002; Snik and Cremers, 2004; Mosnier et al., 2008]. Since 2006, the implant has been used effectively in adult patients with conductive and mixed hearing loss as well as in children [Colletti et al., 2006; Baumgartner et al., 2010; Cremers et al., 2010; Mlynski et al., 2010; Böheim et al., 2012; Huber et al., 2012].

The implanted part of the VSB, the vibrating ossicular replacement prosthesis, consists of a receiver/stimulator,

\section{KARGER}

E-Mail karger@karger.com

www.karger.com/aud (c) 2013 S. Karger AG, Basel

$1420-3030 / 14 / 0191-0001 \$ 39.50 / 0$ 
a conductor link and the floating mass transducer (FMT), converting the electrical signal into mechanical vibrations by means of an electromagnetic transducer. For the classical indication with normal middle-ear anatomy, the FMT is fixed to the long process of the incus [Fisch et al., 2001]. Attachment of the FMT to the stapes or its remnants, the round window (RW) or a variety of coupling elements are alternatives for patients with conductive and mixed hearing loss [Colletti et al., 2006; Streitberger et al., 2009; Baumgartner et al., 2010; Mlynski et al., 2010; Beleites et al., 2011; Böheim et al., 2012; Huber et al., 2012].

The standard surgical approach is a mastoidectomy and a posterior tympanotomy. The facial recess must be widely exposed to obtain an overview of the ossicular chain and to insert the FMT (length $2.3 \mathrm{~mm}$, diameter $1.8 \mathrm{~mm}$ ) as well as to use the opened crimping forceps. The FMT is fixed at the long process of the incus, in such a manner that the axis of the FMT is parallel to the axis of the piston component of stapes motion. Sufficient space in the middle-ear cavity is required to ensure that FMT motion is not impeded by nonvibratory structures such as the promontory, the pyramidal eminence or the tympanic membrane. Firm attachment of the FMT to the long incus process is crucial for the performance of the device [Fisch et al., 2001]. The attachment must be firm to ensure maximal energy transfer but without being overly tight to avoid necrosis. However, crimping of the FMT to the long incus process may not always produce optimal attachment. This has been discussed as a probable cause for a variation in outcome [Snik et al., 2001]. Several authors have used bone cement to optimize attachment in cases of unsuccessful fixation [Lenarz et al., 2001; Snik and Cremers, 2004]. Three cases of incus necrosis have been described several years after VSB implantation [Verhaegen et al., 2012].

Numerous technical solutions for active middle-ear implants use the incus body for transfer of vibratory energy [Maniglia et al., 1994; Fredrickson et al., 1995; Zenner and Leysieffer, 1997]. Incus body stimulation provides appropriate gain as a function of degree of hearing loss, indicating that these devices are a viable treatment for moderate-to-severe hearing loss [Jenkins et al., 2004; Zenner and Rodriguez Jorge, 2010; Klein et al., 2012].

The aim of this study was to determine if attachment of the FMT to the short incus process provides efficient vibratory energy comparable to stimulation when the FMT is attached to the long incus process. This was ascertained by measuring the vibration responses of the stapes footplate and RW membrane in response to acoustic stimulation in the ear canal and to electromechanical stimulation with the FMT at the long and short processes of the incus.

\section{Materials and Methods}

\section{Temporal Bones}

Five left human temporal bones were extracted from human cadavers at the time of autopsy, within $48 \mathrm{~h}$ postmortem, using an oscillating bone saw and subsequently placed in a deep freezer at $-18^{\circ} \mathrm{C}$. The temporal bone specimens were obtained from the Department of Pathology, University of Würzburg, following appropriate guidelines and procedures for obtaining and using human tissue. On the day of the experiment, the temporal bone was allowed to thaw at room temperature and was then immersed in saline solution ( $0.9 \%$ ) for about $3 \mathrm{~h}$ before beginning the experiments. Experiments were performed within 3 months postmortem.

After removal of connective tissue, a subtotal mastoidectomy and a posterior tympanotomy were performed. The RW niche was exposed by drilling promontory overhangs until the RW membrane was identified in its full circumference (fig. 1a). Middle-ear ligaments and muscles as well as the facial nerve with its chorda tympani were not sectioned. Specimens were firmly mounted in a holding block. The prepared bones were kept moist by repeated flushing with saline solution $(0.9 \%)$ to prevent exsiccation. Experiments were performed in a temperature-controlled laboratory $\left(21 \pm 1^{\circ} \mathrm{C}\right)$.

Dissections were performed by experienced otosurgeons (S.P.S. and R.M.), and the FMT was crimped onto the incus by a surgeon experienced with this surgery in patients (R.M.). All ears appeared anatomically normal, as ascertained by light-microscopic examination.

\section{Measurement Setup}

The measurement setup has been described in detail elsewhere [Schraven et al., 2011]. In short, a laser Doppler vibrometer (LDV) system (Model OFV 302, Polytec GmbH, Waldbronn, Germany), focused on a 45 - to $63-\mu \mathrm{m}$-diameter reflective glass microbead (specific gravity 2.5; P-Retro, Polytec) placed on the stapes footplate and on the RW membrane, was used for velocity measurements. Only a single bead was placed on each structure and was located near its centre. LDV measurements of the stapes footplate and the RW membrane were performed sequentially.

To guarantee reproducible positioning of the temporal bone and to control the relative position of the temporal bone and its azimuthal angle with respect to the LDV beam, the specimen was placed on an adjustment-stage setup composed of an upper section (3-axis goniometric adjustment stage) and a lower section (rotary table and an xyz-translational stage) [Schraven et al., 2011; Schraven et al., 2012]. The spatial angle between the surface of the stapes footplate and the LDV laser beam was $40-60^{\circ}$. Velocities are presented uncorrected for spatial measurement angle.

\section{Stimulus Generation and Acquisition}

Signal generation and data acquisition were performed using a 16-bit AD/DA card (PCI-MIO-16E-1, National Instruments, Austin, Tex., USA). The sampling rate was set to $80,000 / \mathrm{s}$ and block length to 2048 . The card was controlled by custom-made software. 

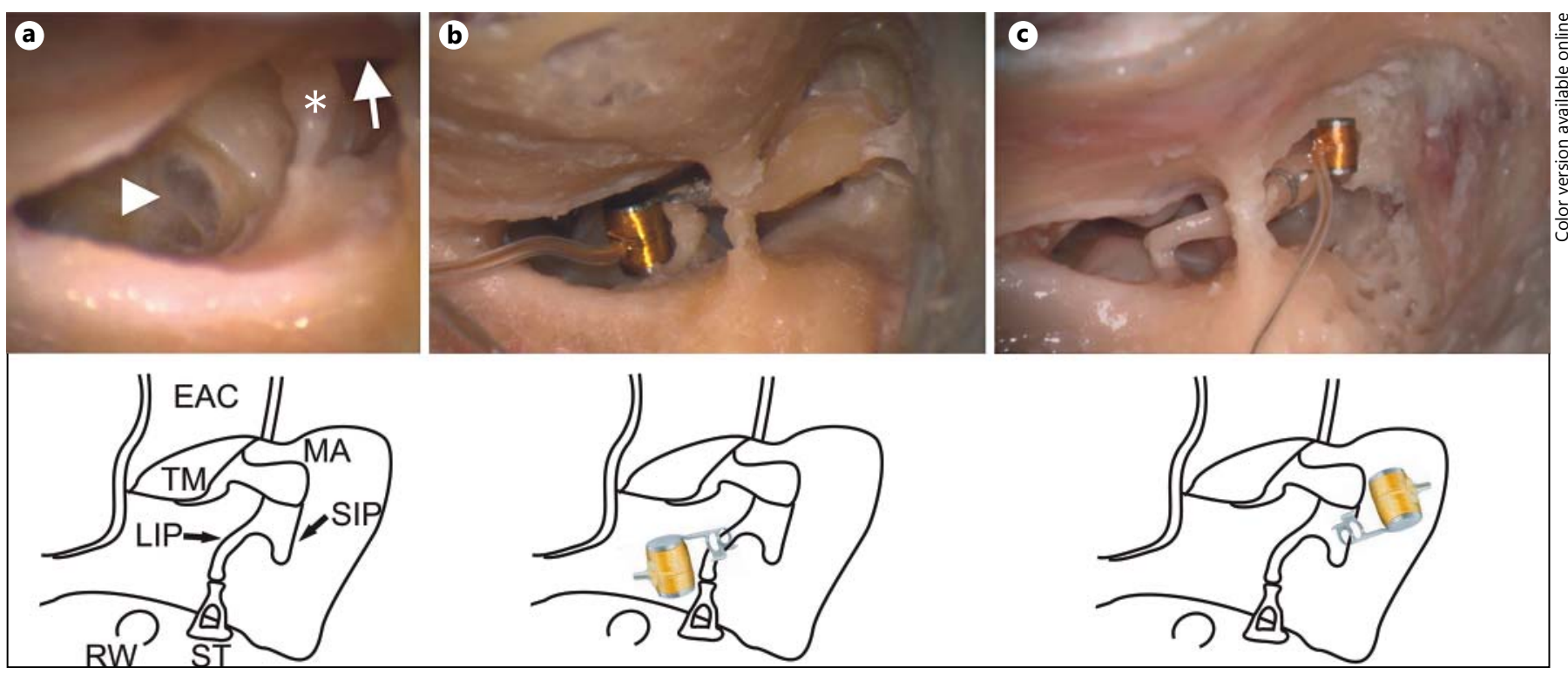

Fig. 1. a View of the stapes (asterisk), the long process of the incus (arrow) and the RW (arrowhead) through the facial recess. b Application of the FMT at the long incus process (standard application). c Application of the FMT at the short incus process. The sketches are designed to illustrate the position and orientation of the FMT relative to the incus and the stapes. EAC = External auditory canal; $\mathrm{TM}=$ tympanic membrane; $\mathrm{MA}=$ malleus; $\mathrm{LIP}=$ long incus process; $\mathrm{SIP}=$ short incus process; $\mathrm{ST}=$ stapes.
Frequency responses were obtained using a multitone complex with equal amplitude and random, uniformly distributed phase. The multitone complex covered a frequency range of $0.2-20 \mathrm{kHz}$, with 9.1 lines per octave. All measurements were averaged 300 times. The sensitivity of the LDV velocity decoder was set to $1 \mathrm{~V} /$ $\mathrm{mm} / \mathrm{s}$. The LDV signal was fed to the first input channel of the $\mathrm{AD} /$ DA card.

In the case of acoustic stimulation, the stimulus was connected to one of the two speakers of an otoacustic emission insert probe (ER-10C, Etymotic Research, Elk Grove, Ill., USA). The amplitude of the multitone complex was set to $0.35 \mathrm{~V}_{\text {rms }}$ (Volt root mean square) per spectral line, corresponding to approximately $60 \mathrm{~dB}$ sound pressure level. The output of the probe's microphone was fed to the second input channel of the AD/DA card. The sound pressure was computed from the voltage using the microphone sensitivity at $1 \mathrm{kHz}$. The vibration responses of the stapes footplate and the RW membrane to sound stimulation were then computed as velocity relative to sound pressure. The signal-to-noise ratio (SNR) was $>10 \mathrm{~dB}$ for stimulus frequencies below $1 \mathrm{kHz}$ and $>20$ $\mathrm{dB}$ at higher frequencies.

In the case of electromechanical stimulation with the FMT, the voltage stimulus was connected directly to the FMT, with the amplitude set to $0.035 \mathrm{~V}_{\text {rms }}$ per spectral line, ensuring that the FMT operated within its linear range, at the same time providing sufficient SNR. Linearity was ascertained in pilot experiments using single-tone stimuli at $0.8,1.6,3.2$ and $6.4 \mathrm{kHz}$, where it was found that total harmonic distortion was more than $35 \mathrm{~dB}$ below the fundamental for FMT stimulus voltages at and below $0.07 \mathrm{~V}_{\mathrm{rms}}$. The vibration responses of the stapes footplate and the RW membrane are presented as velocity relative to $1-\mathrm{V}_{\text {rms }}$ driving voltage, assuming linearity. SNR was $>6 \mathrm{~dB}$ for stimulus frequencies below $1 \mathrm{kHz}$ and $>20 \mathrm{~dB}$ at higher frequencies.
Velocity was defined as positive for motion into the cochlea. Polarity of the voltage driving the FMT was connected as specified by the manufacturer.

\section{Measurement Procedure}

A series of 5 measurements at the stapes footplate and the RW membrane was performed in each temporal bone. Acoustic control measurements were performed to check for a proper soundinduced middle-ear transfer function and to control for possible mechanical alterations of the middle ear induced by the surgical application of the FMT.

Firstly, acoustic baseline measurements of the middle ear were obtained. The external auditory canal was occluded by an otoacustic emission insert probe (ER-10C, Etymotic Research). The distance between the speaker outlet and the tympanic membrane was estimated to be $8-10 \mathrm{~mm}$. The vibration of the stapes footplate and the RW membrane in response to the acoustic stimulus was then measured with the LDV.

Secondly, the FMT of a VSB (MED-EL, Innsbruck, Austria) was firmly crimped to the long incus process (fig. 1b), and the vibration measurements of the stapes footplate and the RW membrane in response to the acoustic stimulation were repeated to check for possible alterations of the middle ear due to surgical placement of the FMT.

Thirdly, the vibrations of the stapes footplate and the RW membrane were measured in response to stimulation by the FMT.

Fourthly, the FMT was removed from the long incus process and attached to the short incus process (fig. 1c). The orientation of the FMT was adjusted to be approximately perpendicular to the plane of the stapes footplate as estimated by the surgeon. Again, to check for surgical alterations of the middle ear, the sound-induced vibration response of the stapes footplate and the RW membrane was measured. 


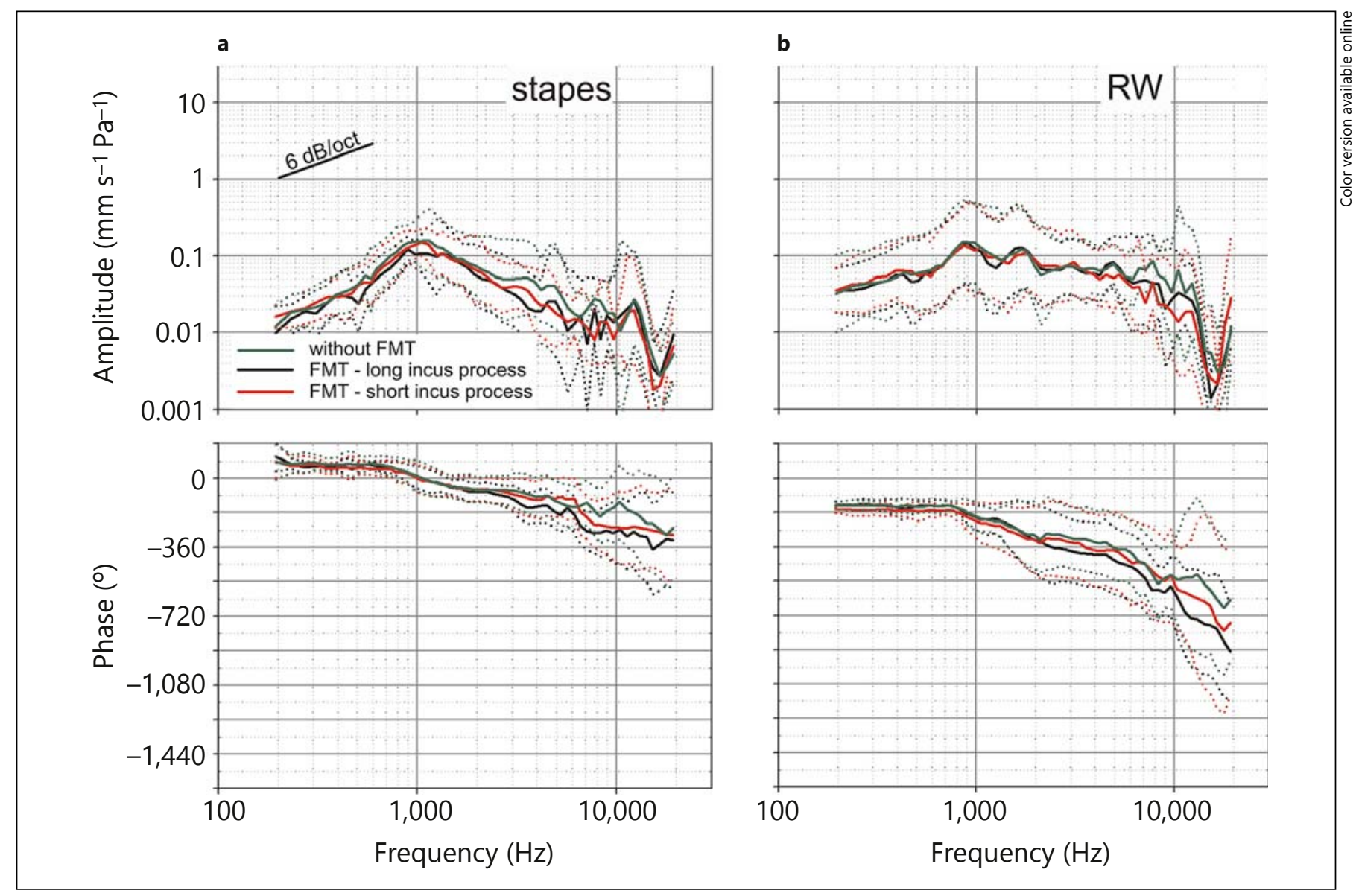

Fig. 2. Mean velocity responses of the stapes footplate (a) and RW membrane (b) for acoustic stimulation. Stimulation is performed by a closed-field method in the ear canal. Means (solid lines) and standard deviations (dotted lines) are from 5 fresh

Fifthly, vibration measurements of the stapes footplate and the RW membrane for stimulation of the short incus process by the FMT were performed.

A brand new FMT was used for every temporal bone to avoid wearing out the attachment clip and also to more accurately simulate the clinical situation. The position of the sound source was not altered during the course of the experiments. In other words, the ear canal was closed for both the acoustical and the electromechanical experiments. The FMT cable was hanging without tension in the free space between the FMT and voltage source, partially lying on bone in the mastoid cavity (fig. 1b, c).

\section{Results}

\section{Acoustic Stimulation}

Figure 2a shows velocity amplitude and phase of the stapes footplate in response to acoustic stimulation of the temporal bones. Notice the similarity of the responses for the 3 incus conditions. The straight line in the upper left corner of a with a slope of $6 \mathrm{~dB} /$ oct has been inserted as an optical guideline.

tympanic membrane. Velocity is given relative to sound pressure. Shown are the mean responses (solid lines) and the standard deviations (dotted lines) for the baseline measurement (without FMT, gray line) and for the control measurements after attachment of the FMT to the long (black line) and the short (red line) process of the incus, respectively. Salient features for all 3 measurement conditions can be collectively described as follows. The low-frequency amplitude response increases with stimulus frequency up to $1 \mathrm{kHz}$, where it reaches a maximum amplitude of $0.1-0.15 \mathrm{~mm} / \mathrm{s} / \mathrm{Pa}$; the standard deviation is typically $4 \mathrm{~dB}$ up to $1 \mathrm{kHz}$. This low-frequency region can be approximated by two line segments, one with a slope of approximately $6 \mathrm{~dB} /$ oct up to about $0.4 \mathrm{~Hz}$ and the other of approximately $15 \mathrm{~dB}$ /oct up to a resonance peak at $1 \mathrm{kHz}$. Above $1 \mathrm{kHz}$, the amplitude response decreases to $0.01-0.02 \mathrm{~mm} / \mathrm{s} / \mathrm{Pa}$ at $7 \mathrm{kHz}$ with a slope of approxi- 


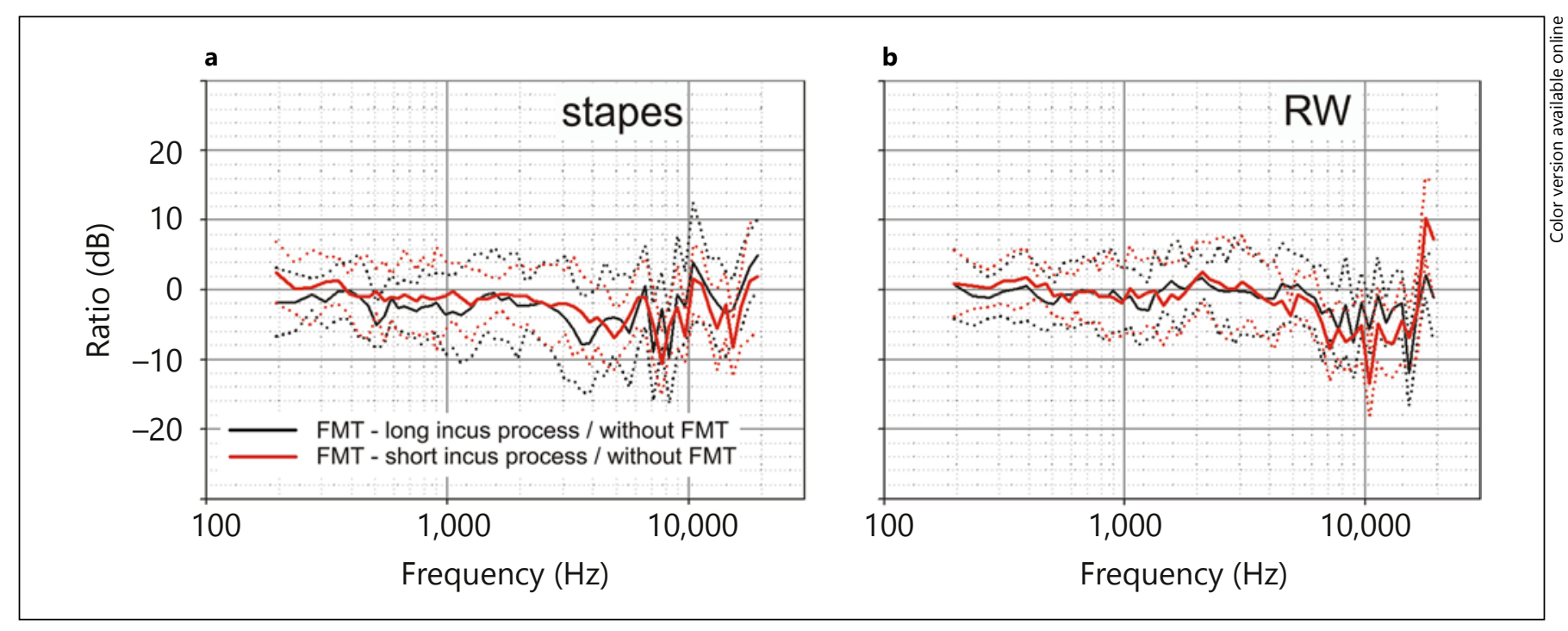

Fig. 3. Mean sound-induced amplitude responses for the FMT attached to an incus process relative to the amplitude response without FMT attachment. Responses are for the stapes footplate (a) and the RW membrane (b). Means (solid lines) and standard deviations (dotted lines) are from the same data as in figure 2, but calculated

mately $-8 \mathrm{~dB} /$ oct; the standard deviation is typically $9 \mathrm{~dB}$ for $1-7 \mathrm{kHz}$. From $7 \mathrm{kHz}$ to the highest stimulus frequency of $20 \mathrm{kHz}$, the amplitude response can be described as that of an amplitude resonance tuned to about $12 \mathrm{kHz}$, which is superimposed on a decreasing amplitude response with a slope of approximately $-8 \mathrm{~dB} /$ oct.

The low-frequency phase response asymptotes to approximately $90^{\circ}$, concomitant with the low-frequency amplitude slope of $6 \mathrm{~dB} /$ oct, which in turn are consistent with compliant controlled motion at low frequencies. The standard deviation is typically $50^{\circ}$ at low frequencies. At $7 \mathrm{kHz}$, the phase accumulates a lag of $210-360^{\circ}$ with respect to the low-frequency asymptote; the standard deviation is typically $120^{\circ}$ at this frequency. At $20 \mathrm{kHz}$, the total phase lag is $350-420^{\circ}$; the standard deviation is typically $260^{\circ}$ at this frequency. The speed of sound in air accounts for $71^{\circ}$ and $208^{\circ}$, respectively, of these phase delays at 7 and $20 \mathrm{kHz}$. After subtracting the acoustic delay of $23 \mu \mathrm{s}$ for an 8 - $\mathrm{mm}$ distance from the sound source to the tympanic membrane, the amplitude and phase responses are mutually consistent, based on minimum phase theory. In this sense, the resonance at $12 \mathrm{kHz}$ almost certainly arises from resonance in the sound field (8 $\mathrm{mm}$ corresponds to a quarter-wavelength frequency of $10.8 \mathrm{kHz})$.

Taken together, the results in figure $2 \mathrm{a}$ suggest that the application procedure and the presence of the FMT - ei- by averaging the ratios from the individual specimens. Apart from minor differences in narrow frequency bands in the stapes responses (at $3.5 \mathrm{kHz}$ for the long process and $5 \mathrm{kHz}$ for the short process), changes due to attachment of the FMT to either the long or the short incus process disappear in the interindividual variation. ther on the long or the short incus process - do not have a significant effect on the mean amplitude or phase of the stapes footplate in response to acoustic stimulation. This conclusion is substantiated by the mean amplitude ratio data in figure $3 \mathrm{a}$, where acoustic stimulation motion in the presence of the FMT is expressed relative to motion in the absence of the FMT. The mean is estimated as the average of the amplitude ratios, expressed in decibels, obtained from the 5 individual temporal bones. At all stimulus frequencies, the mean ratio was not significantly different from $0 \mathrm{~dB}$, again implying that the FMT does not significantly affect the stapes footplate response to acoustic stimulation.

The same is concluded from the corresponding acoustically induced RW membrane responses presented in figures $2 \mathrm{~b}$ and $3 \mathrm{~b}$. These responses were measured directly after the respective stapes footplate response measurements. RW amplitudes are generally higher than stapes amplitudes by a factor of 1.5-3.0 up to $10 \mathrm{kHz}$. The higher amplitudes are evidenced as smaller low- and highfrequency amplitude slopes, whilst maintaining the resonance maximum near $1 \mathrm{kHz}(0.14 \mathrm{~mm} / \mathrm{s} / \mathrm{Pa}$ at $0.9 \mathrm{kHz})$; namely, the slope is $3 \mathrm{~dB} /$ oct for $0.2-0.6 \mathrm{kHz}$ and $-3 \mathrm{~dB} /$ oct for $1-8 \mathrm{kHz}$. The phase responses show the expected counterphasic motion relative to stapes motion and are independent of frequency up to approximately $0.8 \mathrm{kHz}$. Although the standard deviations of the amplitudes and 


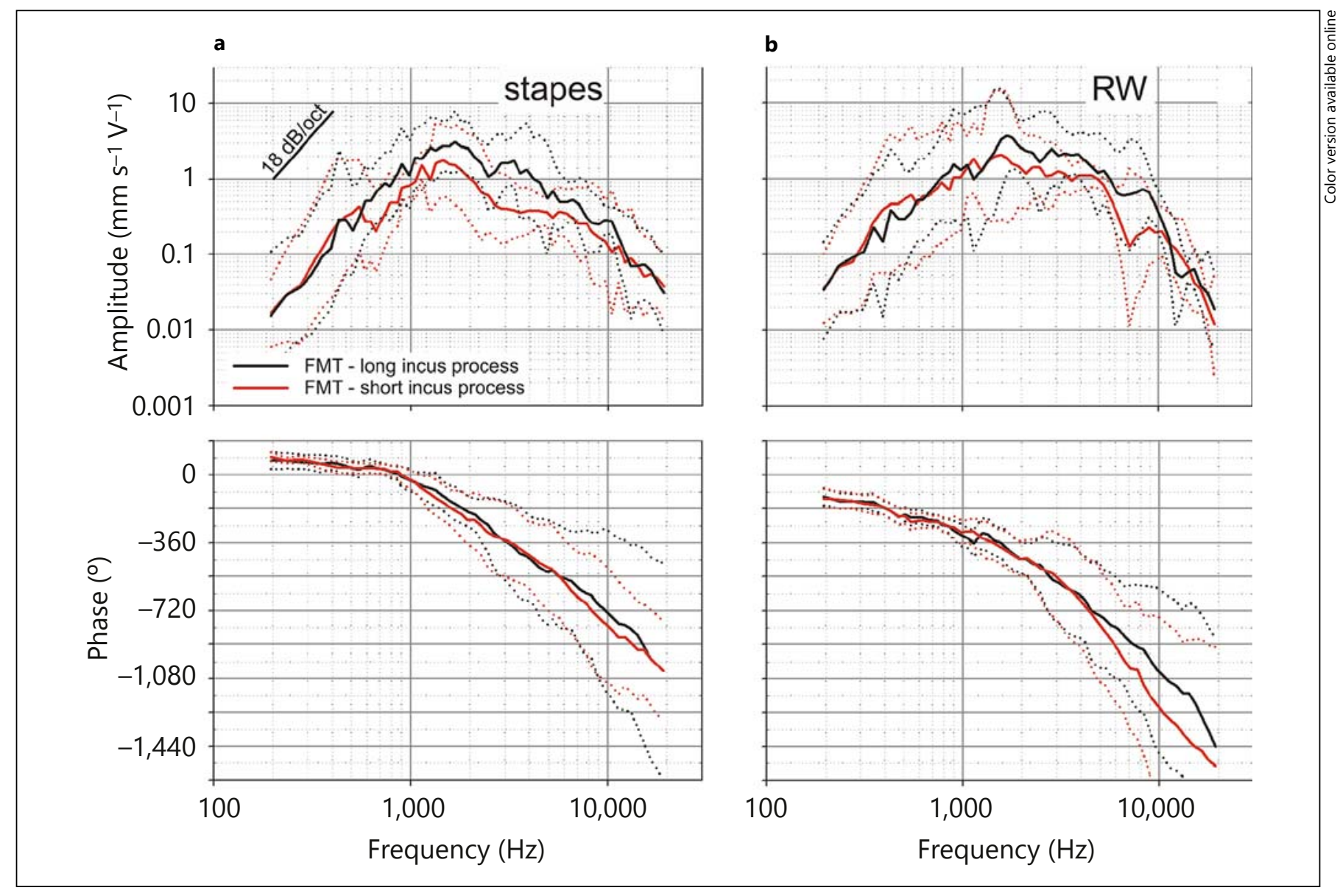

Fig. 4. Mean velocity responses of the stapes footplate (a) and RW membrane (b) for electromechanical stimulation with the FMT attached to the long incus process or the short incus process. Velocity amplitudes are given relative to $1 \mathrm{~V}$, by linearly scaling from the stimulus amplitude of $0.035 \mathrm{~V}_{\text {rms }}$ per spectral line. Means (sol-

phases are generally larger than those for the stapes responses (e.g. approximately $7 \mathrm{~dB}$ instead of $4 \mathrm{~dB}$ at low frequencies), the mean responses for the 3 incus conditions show even greater similarity than for the stapes responses, particularly with regard to the amplitude responses, where there is near superposition up to stimulus frequencies as high as $6 \mathrm{kHz}$. The larger mean differences between the stapes responses for the 3 incus conditions, compared with those for the RW, is possibly due to the fact that the stapes footplate is known to have significant non-piston-like motion at high frequencies [Guinan and Peake, 1967; Decraemer et al., 2007; Sim et al., 2010], but nevertheless the middle-ear pressure gain as a function of frequency is known to be relatively smooth [Decraemer et al., 2007]. In this sense, the RW responses - being perhaps a more direct measure of intracochlear fluid dynam- id lines) and standard deviations (dotted lines) are from the 5 fresh temporal bones used for acoustical stimulation in figure 2 . Notice the similarity of the responses for the 2 incus stimulus conditions. The straight line in the upper left corner of a with a slope of $18 \mathrm{~dB} /$ oct has been inserted as an optical guideline.

ics - provide even greater confidence in the conclusion that the presence of the FMT has an insignificant effect on cochlear input.

\section{Electromechanical Stimulation}

Stimulating the middle ear with the FMT at the long incus process reflects the clinical practice for using the FMT. Therefore, this coupling method is defined here as standard coupling.

Figure 4a shows the mean velocity amplitude and phase of the stapes footplate in response to electromechanical stimulation for the FMT attached to the long (black line) and short (red line) incus processes, respectively. Velocity is given relative to FMT voltage. For both measurement conditions, the low-frequency amplitude response increases with a slope of approximate- 


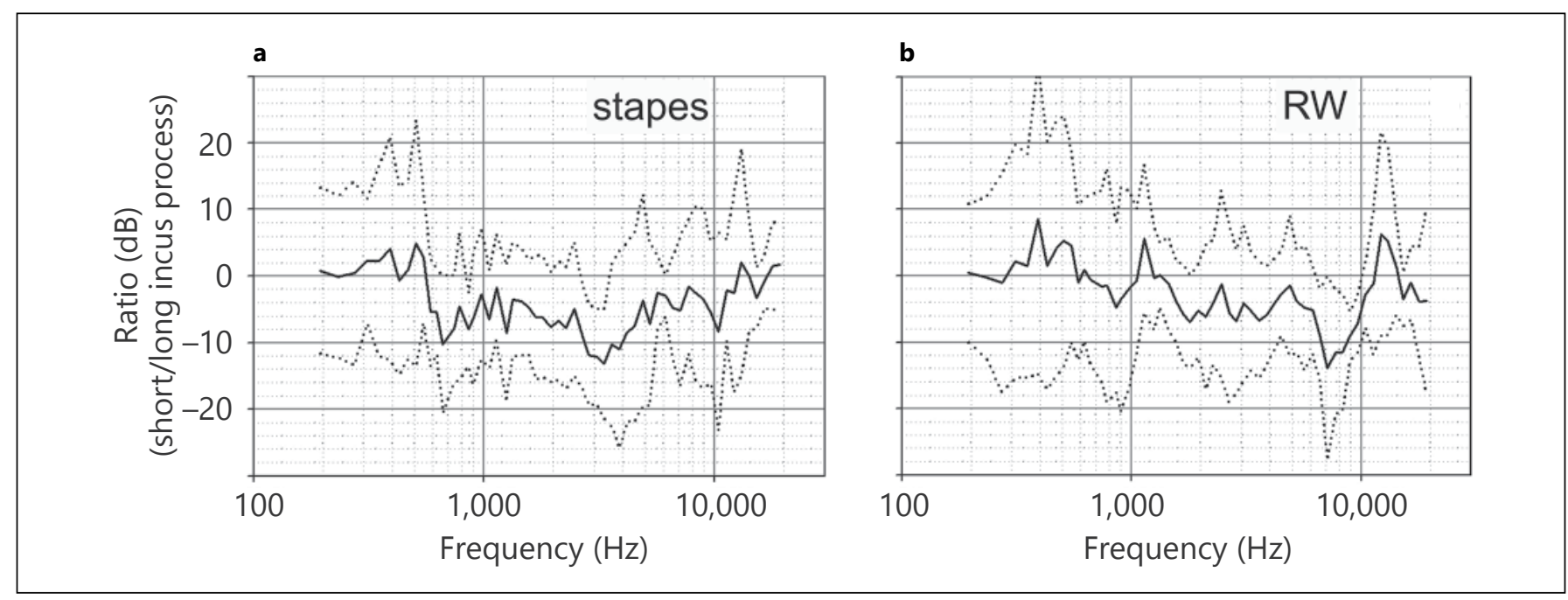

Fig. 5. Mean electromechanically induced amplitude response for the FMT attached to the short incus process relative to the amplitude response for the FMT attached to the long incus process. Responses are for the stapes footplate (a) and the RW membrane (b). Means (solid lines) and standard deviations (dotted lines) are from the same data as in figure 4 , but calculated by averaging the ratios

ly $18 \mathrm{~dB} /$ oct, attaining a maximum amplitude of $1.3-2.5$ $\mathrm{mm} / \mathrm{s} / \mathrm{V}$ at approximately $1.3 \mathrm{kHz}$; the standard deviation is typically $12 \mathrm{~dB}$. Above $1.3 \mathrm{kHz}$, the amplitude response decreases with a (mild) slope of approximately $-6 \mathrm{~dB} /$ oct up to $10 \mathrm{kHz}$, where its amplitude is $0.1-0.3$ $\mathrm{mm} / \mathrm{s} / \mathrm{V}$. The standard deviation is typically $9 \mathrm{~dB}$ for $1.3-10 \mathrm{kHz}$ and, as such, similar to that for the low-frequency region. The amplitude response decreases rapidly above $10 \mathrm{kHz}$ with a slope of $-18 \mathrm{~dB} /$ oct. There appears to be a tendency for slightly decreased amplitudes for short-process fixation at frequencies above $0.6 \mathrm{kHz}$. However, when the short-process amplitude response is expressed relative to the long-process amplitude response for individual preparations and averaged, on a decibel scale, to form the mean relative response for the population (fig. 5a), the ratio is significantly different from $0 \mathrm{~dB}$ only in a narrow band of frequencies, namely for $2.9-3.3 \mathrm{kHz}$, the difference ranging from $-12 \mathrm{~dB}$ $(\mathrm{p}=0.020)$ to $-13 \mathrm{~dB}(\mathrm{p}=0.0245)$.

The low-frequency phase response of the stapes footplate below $0.6 \mathrm{kHz}$ asymptotes to approximately $90^{\circ}$. The standard deviation is typically $50^{\circ}$ at these low frequencies and, therefore, similar to the standard deviation for acoustic stimulation. At $10 \mathrm{kHz}$, the phase accumulates a lag of $810-890^{\circ}$ with respect to the low-frequency asymptote. The standard deviation for the short-incus process is slightly less than that for the long-incus pro- from the individual specimens. Apart from narrow frequency bands at $3 \mathrm{kHz}$ (stapes footplate) and $9 \mathrm{kHz}$ (RW membrane), the ratio is not significantly different from $0 \mathrm{~dB}$, implying that attaching the FMT to the short incus process instead of to the long incus process (the standard attachment) produces no detectable difference in the amplitude response. cess, amounting to 280 and $400^{\circ}$, respectively, at $10 \mathrm{kHz}$. The phase responses practically superimpose up to $6 \mathrm{kHz}$.

The salient features of the RW membrane responses for the different incus stimulation conditions (fig. $4 \mathrm{~b}$, $5 b)$ are similar to those for the stapes footplate. However, the RW amplitudes are higher by a factor of 1-3, the difference being mainly due to the low-frequency slope being $12 \mathrm{~dB} /$ oct rather than the $18 \mathrm{~dB} /$ oct found for the stapes footplate. The slope from 1.4 to $10 \mathrm{kHz}$ is $-6 \mathrm{~dB} /$ oct, as also found for the stapes footplate. The standard deviation is typically $12 \mathrm{~dB}$, as also found for the stapes footplate. Referring to the mean ratio amplitude in figure $5 \mathrm{~b}$, there was no significant difference between the amplitude responses for the 2 incus placements, except in a narrow band of frequencies, namely for $8.3-9.7 \mathrm{kHz}$, where the short-incus amplitude was smaller by $-7 \mathrm{~dB}(\mathrm{p}=0.005)$ to $-10 \mathrm{~dB}(\mathrm{p}=0.043)$, respectively. The RW phase responses show the expected counterphasic motion relative to stapes motion; the accumulated phase lag at $10 \mathrm{kHz}$ is $930-1,140^{\circ}$ relative to the low-frequency asymptote, where phase rotation is larger for the short-process placement. However, the phases well-nigh superimpose up to $5 \mathrm{kHz}$, and the differences at higher frequencies are not statistically significant.

In summary, the results in figures 4 and 5 suggest that the electromechanically driven velocity responses of the 
stapes footplate and the RW membrane are not significantly affected by fixing the FMT to the short incus process rather than to its standard position on the long incus process.

\section{Discussion}

\section{Surgical Issues}

Since its introduction in 1996, the standard surgical approach for positioning the VSB is a subtotal mastoidectomy combined with a posterior tympanotomy. The results for hearing restoration in sensorineural hearing loss and recently in mixed and conductive hearing loss are reliable and stable in the long term [Fisch et al., 2001; Lenarz et al., 2001; Todt et al., 2002; Mosnier et al., 2008; Streitberger et al., 2009; Böheim et al., 2012; Huber et al., 2012]. However, the surgical approach and the variability in audiological outcome as well as the benefit when compared to conventional hearing aids are controversial, especially with regard to the classical application at the long incus process [Lenarz et al., 2001; Snik et al., 2001; Junker et al., 2002; Schmuziger et al., 2006; Truy et al., 2006; Bruschini et al., 2009].

Subtotal mastoidectomy and posterior tympanotomy have the potential for facial nerve injury. Although there is no report of facial nerve injury with VSB implantation, the incidence of temporary or permanent facial nerve palsy in cochlear implantation using this surgical approach has been reported to be between 0.3 and 2.2\% [Cohen et al., 1991; Brito et al., 2012]. Moreover, one suspects that there are also unreported cases of injury with VSB surgery. The dimensions of the FMT necessitate large opening of the facial recess and exposure of the chorda tympani. The incidence of severed chorda tympani has been reported to be $1.6 \%$ and that of taste disturbance $6.5 \%$ after VSB surgery; the symptoms resolve in approximately $50 \%$ of patients [Sterkers et al., 2003; Truy et al., 2006]. Using these potential risks as an argument, transcanal surgical approaches have been described as an alternative [Truy et al., 2006; Bruschini et al., 2009].

Another surgical issue is the fixation of the FMT at the long incus process. Firm attachment of the FMT to the long incus process is crucial for optimal performance of the device [Fisch et al., 2001]. Limited access through the posterior tympanotomy and compromised crimping due to the conical shape of the long incus process have been discussed as probable causes for a variation in outcome [Snik et al., 2001]. There are at least 3 reported cases of incus necrosis [Verhaegen et al., 2012], presumably because of overtightening of the FMT clip onto the incus. Several authors have tried using bone cement to facilitate reliable FMT attachment when experiencing difficulties fixing at the long incus process [Lenarz et al., 2001; Snik and Cremers, 2004]. However, this technique is not normally used because audiometric results with bone cement show no significant benefit for these patients [Snik and Cremers, 2004]. Another reason for variable results might be the relatively large dimensions of the FMT, which might lead to reduced middle-ear aeration and limit audiological outcome. Promising results with placement of the FMT at other stimulatory sites in the middle ear, as well as the benefits of incus body stimulation in other implantable hearing aid solutions, were the stimuli for our investigation of the effect on velocity responses of fixation of the FMT at the short incus process. Velocity responses were evaluated not only at the stapes footplate, as a measure of the input to the cochlea, but also at the RW membrane as a (rough) indicator of intracochlear fluid dynamics.

\section{Acoustic Responses}

The present control data for sound-induced stapes velocity in the absence of the FMT (fig. 2a) fall well within the 'normal' criterion range defined in earlier temporal bone studies, collated and summarized in Rosowski et al. [2007]. That is, within the realm of temporal bone studies, we have confidence in the viability of the preparations. With respect to in vivo characteristics, it has been reported [Huber et al., 2012] that the main difference between stapes responses in vivo and in vitro is that low-frequency in vivo amplitudes $(0.5-1.2 \mathrm{kHz})$ are lower than temporal bone amplitudes below about $1.2 \mathrm{kHz}$, the difference increasing at low frequencies (approx. $10 \mathrm{~dB}$ at $0.5 \mathrm{kHz}$ ). This point should be kept in mind when interpreting the in vitro low-frequency FMT responses because it suggests that the middle ear presents greater stiffness in vivo compared with the (viable) temporal bone situation. Conversely, at high frequencies, where middle-ear inertia becomes increasingly important, the temporal bone experiments are expected to closely mimic the in vivo situation. For example, in this respect, by studying the effect of attaching the FMT to the short incus process, to a first approximation we have effectively examined the effect of placing the FMT closer to the center of mass of the middle ear than that which is done normally.

Within the interindividual variation of control responses, the presence of the FMT - on either the long or
Schraven/Dalhoff/Wildenstein/Hagen/ Gummer/Mlynski 
the short process of the incus - did not significantly affect the velocity responses of either the stapes or the RW to acoustic stimulation up to at least $6 \mathrm{kHz}$ (fig. 2, 3). This finding is similar to that observed (for stapes acoustic responses) by Rosowski et al. [2007; their fig. 4B] in the case of control experiments with their commercially obtained electromechanical transducer [denoted by them as implantable middle-ear hearing device A (IMEHD-A); the manufacturer was not identified, but as explained below it was probably an FMT]. The present results and those of Rosowski et al. [2007] are at variance with the results of Needham et al. [2005], who report a statistically significant loading effect of the FMT (MED-EL) which systematically increases with frequency and which for the usual incus location amounts to $8 \mathrm{~dB}$ at the highest measured frequency of $5 \mathrm{kHz}$ (their fig. 7, position 1). Apart from methodological issues, we have no concrete explanation for the discrepancy. For example, perhaps the difference is simply due to how the cable between the voltage source and the FMT was supported. In this respect, using a physical mechanical model of the middle ear, Stieger et al. [2007] have shown that, depending on the amount of crimping, an additional load of $150 \mathrm{mg}$ on the cable, placed $4 \mathrm{~mm}$ from the FMT, can produce high-frequency $(4-10 \mathrm{kHz}$ ) loss of up to $23 \mathrm{~dB}$ (their fig. 5C).

\section{Electromechanical Responses}

Irrespective of whether the FMT is attached to the long or short incus process, the stapes velocity response to FMT stimulation can be summarized as follows: (1) a low-frequency $(0.2-1.3 \mathrm{kHz})$ amplitude slope of $18 \mathrm{~dB} /$ oct, (2) maximum amplitude $(1.3-2.5 \mathrm{~mm} / \mathrm{s} / \mathrm{V})$ at approximately $1.3 \mathrm{kHz},(3)$ a high-frequency $(1.3-10 \mathrm{kHz})$ amplitude slope of $-6 \mathrm{~dB} /$ oct, (4) a low-frequency phase asymptote of $90^{\circ}$ and (5) an accumulated phase lag up to $10 \mathrm{kHz}$ of $810-890^{\circ}(2.25-2.5$ cycles $)$. The low-frequency amplitude slope (18 dB/oct) and phase asymptote $\left(90^{\circ}\right)$ are expected theoretically when the FMT drives a compliant load (unpublished modelling). Comparing acoustic and FMT-driven amplitudes in figures $2 \mathrm{a}$ and $4 \mathrm{a}$, respectively, the FMT stimulus voltage of $0.035 \mathrm{~V}_{\text {rms }}$ per spectral line produces a stapes velocity at $1 \mathrm{kHz}$ equivalent to $85 \mathrm{~dB}$ sound pressure level. The standard deviation of the low-frequency amplitudes was greater for FMT than for acoustic stimulation, being $12 \mathrm{~dB}$ rather than $4 \mathrm{~dB}$. The reason for this difference is not yet known; this topic requires further experimental and theoretical investigation.

Perhaps the most comprehensive data set, allowing comparisons of both amplitude and phase responses with our data, is that of Rosowski et al. [2007]. However, the manufacturer of their commercially obtained implantable middle-ear hearing device, designated by them with the acronym IMEHD-A, was not identified. Rosowski et al. [2007] reported an amplitude response increasing with slope of approximately $18 \mathrm{~dB}$ /oct between 0.15 and $0.5 \mathrm{kHz}$ and a resonance frequency of $2 \mathrm{kHz}$ with an amplitude maximum of $3 \mathrm{~mm} / \mathrm{s} / \mathrm{V}$; the low-frequency phase asymptote was 0.25 cycles and the total phase accumulation was 2.5 cycles up to $10 \mathrm{kHz}$. That is, the present results concur with those of Rosowski et al. [2007]. However, we did not find significant local minima on the low-frequency flank of the mean amplitude response as observed by Rosowski et al. [2007; at 0.8 and $1.3 \mathrm{kHz}$ on their fig. 6]. Park et al. [2011] assert that peaks and dips sometimes found in the FMT (displacement) response of the stapes might be due to imperfect clamping of the FMT onto the long incus process. In any case, the IMEHD-A device used by Rosowski et al. [2007] was presumably an FMT, because the low-frequency amplitude slope of $18 \mathrm{~dB} /$ oct is characteristic of FMT responses [Stieger et al., 2007; Park et al., 2011; Shimizu et al., 2011], and other commercial devices available at that time were not based on this principle. (Depending on the report, the low-frequency amplitude slope lies in the range 15-21 $\mathrm{dB} /$ oct.)

\section{Comparison of FMT Responses for the Two Incus Attachment Sites}

The vibration measurements on the stapes footplate and RW membrane revealed that FMT attachment to the short incus process produces essentially the same velocity responses in comparison to the standard coupling to the long incus process (fig. 4, 5). There was a slight tendency for decreased amplitudes for the short incus process placement, typically less than $5 \mathrm{~dB}$. However, differences were only statistically significant in narrow frequency bands, namely up to $13 \mathrm{~dB}$ between 2.9 and 3.3 $\mathrm{kHz}$ for the stapes footplate and $12 \mathrm{~dB}$ between 8.3 and $9.7 \mathrm{kHz}$ for the RW membrane. Moreover, phase responses were well-nigh superimposable up to $5 \mathrm{kHz}$, which covers the frequency range required for speech. Given that the surgical procedure is much less invasive for attachment to the short incus process, it makes sense to investigate this fixation site more closely in the future and to develop a standardized and reliable attachment of the FMT to the short incus process. Although the standard clip at the FMT was sufficient in this proof-of-principle study, it may not be sustainable for a long-term application. 


\section{Conclusion}

Fixation of the FMT to the short incus process showed similar velocity responses of the stapes footplate and the RW membrane when compared to its attachment to the long incus process. The fixation of the FMT to the short incus process may be an efficient alternative surgical approach in patients with moderate-to-severe sensorineural hearing loss. The surgery could be limited to an extended antrotomy.

\section{Acknowledgments}

MED-EL (Innsbruck, Austria) sponsored this study by providing FMTs for experimental use. The authors themselves did not receive any financial support from MED-EL. The experimental setup was financially supported by a grant from the Deutsche Forschungsgemeinschaft (DFG Gu 194/9-1).

\section{References}

Baumgartner WD, Böheim K, Hagen R, Müller J, Lenarz T, Reiss S, Schlögel M, Mlynski R, Mojallal H, Colletti V, Opie J: The vibrant soundbridge for conductive and mixed hearing losses: European multicenter study results. Adv Otorhinolaryngol 2010;69:38-50.

Beleites T, Neudert M, Beutner D, Hüttenbrink $\mathrm{KB}$, Zahnert T: Experience with vibroplasty couplers at the stapes head and footplate. Otol Neurotol 2011;32:1468-1472.

Böheim K, Mlynski R, Lenarz T, Schlögel M, Hagen R: Round window vibroplasty: long-term results. Acta Otolaryngol 2012;132:10421048.

Brito R, Monteiro TA, Leal AF, Tsuji RK, Pinna $\mathrm{MH}$, Bento RF: Surgical complications in 550 consecutive cochlear implantation. Braz J Otorhinolaryngol 2012;78:80-85.

Bruschini L, Forli F, Giannarelli M, Bruschini P, Berrettini S: Exclusive transcanal surgical approach for Vibrant Soundbridge implantation: surgical and functional results. Otol Neurotol 2009;30:950-955.

-Cohen NL, Hoffman RA: Complications of cochlear implant surgery in adults and children. Ann Otol Rhinol Laryngol 1991;100:708-711.

Colletti V, Soli SD, Carner M, Colletti L: Treatment of mixed hearing losses via implantation of a vibratory transducer on the round window. Int J Audiol 2006;45:600-608.

-Cremers CW, O’Connor AF, Helms J, Roberson J, Clarós P, Frenzel H, Profant M, Schmerber S, Streitberger C, Baumgartner WD, Orfila D, Pringle M, Cenjor C, Giarbini N, Jiang D, Snik AF: International consensus on Vibrant Soundbridge ${ }^{\circledR}$ implantation in children and adolescents. Int J Pediatr Otorhinolaryngol 2010;74:1267-1269.

-Decraemer WF, de La Rochefoucauld O, Dong W, Khanna SM, Dirckx JJ, Olson ES: Scala vestibuli pressure and three-dimensional stapes velocity measured in direct succession in gerbil. J Acoust Soc Am 2007;121:2774-2791.

-Fisch U, Cremers CW, Lenarz T, Weber B, Babighian G, Uziel AS, Proops DW, O’Connor AF, Charachon R, Helms J, Fraysse B: Clinical experience with the Vibrant Soundbridge implant device. Otol Neurotol 2001;22:962-972.
Fredrickson JM, Coticchia JM, Khosla S: Ongoing investigations into an implantable electromagnetic hearing aid for moderate to severe sensorineural hearing loss. Otolaryngol Clin North Am 1995;28:107-120.

Guinan JJ, Peake WT: Middle-ear characteristics of anesthetized cats. J Acoust Soc Am 1967;41: 1237-1261.

Huber AM, Mlynski R, Müller J, Dillier N, Holzmann D, Wolframm MD, Hagen R: A new vibroplasty coupling technique as a treatment for conductive and mixed hearing losses: a report of 4 cases. Otol Neurotol 2012;33:613617.

Jenkins HA, Niparko JK, Slattery WH, Neely JG, Fredrickson JM: Otologics Middle Ear Transducer Ossicular Stimulator: performance results with varying degrees of sensorineural hearing loss. Acta Otolaryngol 2004;124:391394.

Junker R, Gross M, Todt I, Ernst A: Functional gain of already implanted hearing devices in patients with sensorineural hearing loss of varied origin and extent: Berlin experience. Otol Neurotol 2002;23:452-456.

Klein K, Nardelli A, Stafinski T: A systematic review of the safety and effectiveness of fully implantable middle ear hearing devices: the $\mathrm{Ca}$ rina and Esteem systems. Otol Neurotol 2012; 33:916-921.

Lenarz T, Weber BP, Issing PR, Gnadeberg D, Ambjørnsen K, Mack KF, Winter M: Vibrant Sound Bridge System. A new kind hearing prosthesis for patients with sensorineural hearing loss. 2. Audiological results. Laryngorhinootologie 2001;80:370-380.

Maniglia AJ, Ko WH, Rosenbaum M, Zhu WL, Werning J, Belser R, Drago P, Falk T, Frenz $\mathrm{W}$ : A contactless electromagnetic implantable middle ear device for sensorineural hearing loss. Ear Nose Throat J 1994;73:78-90.

Mlynski R, Müller J, Hagen R: Surgical approaches to position the Vibrant Soundbridge in conductive and mixed hearing loss. Operative Techniques in Otolaryngology 2010;21:272277.

Mosnier I, Sterkers O, Bouccara D, Labassi S, Bebear JP, Bordure P, Dubreuil C, Dumon T,
Frachet B, Fraysse B, Lavieille JP, Magnan J, Martin C, Meyer B, Mondain M, Portmann D, Robier A, Schmerber S, Thomassin JM, Truy E, Uziel A, Vanecloo FM, Vincent C, Ferrary E: Benefit of the Vibrant Soundbridge device in patients implanted for 5 to 8 years. Ear Hear 2008;29:281-284.

Needham AJ, Jiang D, Bibas A, Jeronimidis G, Fitzgerald O'Connor A: The effects of mass loading the ossicles with a floating mass transducer on middle ear transfer function. Otol Neurotol 2005;26:218-224.

Park I-Y, Shimizu Y, O’Connor KN, Puria S, Cho $\mathrm{J}-\mathrm{H}$ : Comparisons of electromagnetic and piezoelectric floating-mass transducers in human cadaveric temporal bones. Hear Res 2011;272:187-192.

Rosowski JJ, Chien W, Ravicz ME, Merchant SN: Testing a method for quantifying the output of implantable middle ear hearing devices. Audiol Neurotol 2007;12:265-276.

Schmuziger N, Schimmann F, àWengen D, Patscheke J, Probst R: Long-term assessment after implantation of the Vibrant Soundbridge device. Otol Neurotol 2006;27:183-188.

-Schraven SP, Hirt B, Goll E, Heyd A, Gummer AW, Zenner HP, Dalhoff E: Conditions for highly efficient and reproducible round-window stimulation in humans. Audiol Neurotol 2012;17:133-138.

Schraven SP, Hirt B, Gummer AW, Zenner HP, Dalhoff E: Controlled round-window stimulation in human temporal bones yielding reproducible and functionally relevant stapedial responses. Hear Res 2011;282:272-282.

Shimizu Y, Puria S, Goode RL: The floating mass transducer on the round window versus attachment to an ossicular replacement prosthesis. Otol Neurotol 2011;32:98-103.

-Sim JH, Chatzimichalis M, Lauxmann M, Röösli C, Eiber A, Huber AM: Complex stapes motions in human ears. J Assoc Res Otolaryngol 2010;11:329-341.

Snik A, Cremers C: Audiometric evaluation of an attempt to optimize the fixation of the transducer of a middle-ear implant to the ossicular chain with bone cement. Clin Otolaryngol Allied Sci 2004;29:5-9.
10
Audiol Neurotol 2014;19:1-11 DOI: $10.1159 / 000354981$
Schraven/Dalhoff/Wildenstein/Hagen/ Gummer/Mlynski 
-Snik AF, Mylanus EA, Cremers CW, Dillier N, Fisch U, Gnadeberg D, Lenarz T, Mazolli M, Babighian G, Uziel AS, Cooper HR, O'Connor AF, Fraysse B, Charachon R, Shehata-Dieler WE: Multicenter audiometric results with the Vibrant Soundbridge, a semi-implantable hearing device for sensorineural hearing impairment. Otolaryngol Clin North Am 2001;34:373-388.

-Sterkers O, Boucarra D, Labassi S, Bebear JP, Dubreuil C, Frachet B, Fraysse B, Lavieille JP, Magnan J, Martin C, Truy E, Uziel A, Vaneecloo FM: A middle ear implant, the Symphonix Vibrant Soundbridge: retrospective study of the first 125 patients implanted in France. Otol Neurotol 2003;24:427-436.
Stieger C, Bernhard H, Waeckerlin D, Kompis M, Burger J, Haeusler R: Human temporal bones versus mechanical model to evaluate three middle ear transducers. J Rehabil Res Dev 2007;44:407-416.

Streitberger C, Perotti M, Beltrame MA, Giarbini $\mathrm{N}$ : Vibrant Soundbridge for hearing restoration after chronic ear surgery. Rev Laryngol Otol Rhinol (Bord) 2009;130:83-88.

Todt I, Seidl RO, Gross M, Ernst A: Comparison of different vibrant soundbridge audioprocessors with conventional hearing aids. Otol Neurotol 2002;23:669-673.

Truy E, Eshraghi AA, Balkany TJ, Telishi FF, Van De Water TR, Lavieille JP: Vibrant soundbridge surgery: evaluation of transcanal surgical approaches. Otol Neurotol 2006;27:887895.
Verhaegen VJ, Mulder JJ, Cremers CW, Snik AF: Application of active middle ear implants in patients with severe mixed hearing loss. Otol Neurotol 2012;33:297-301.

Zenner HP, Leysieffer H: Active electronic cochlear implants for middle and inner ear hearing loss - a new era in ear surgery. I. Basic principles and recommendations on nomenclature. HNO 1997;45:749-757.

Zenner HP, Rodriguez Jorge J: Totally implantable active middle ear implants: ten years' experience at the University of Tübingen. Adv Otorhinolaryngol 2010;69:7284. 